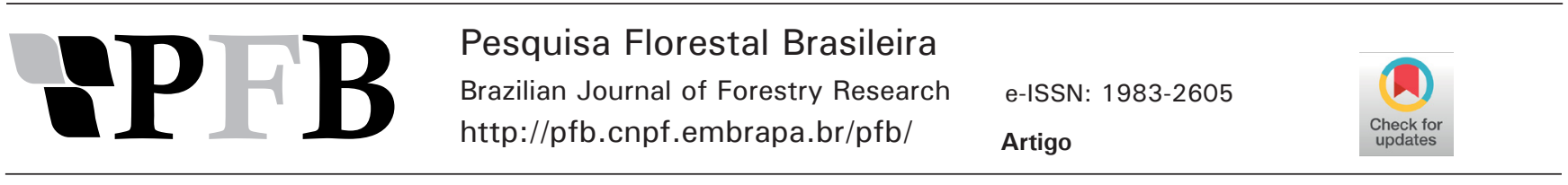

\title{
Deposição de nitrogênio e influência das copas das árvores no efluxo de $\mathrm{C}-\mathrm{CO}_{2}$ no solo
}

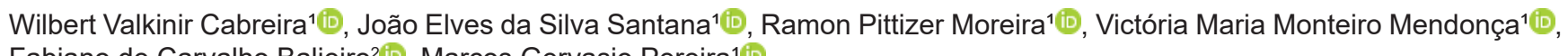
Fabiano de Carvalho Balieiro²(iD), Marcos Gervasio Pereira' ${ }^{1}$ (D)

Universidade Federal Rural do Rio de Janeiro, Km 07, BR-465, CEP 23890-000, Seropédica, RJ, Brasil

${ }^{2}$ Embrapa Solos, Rua Jardim Botânico, 1024, CEP 224960-000, Rio de Janeiro, RJ, Brasil

"Autor correspondente:

wilbertvalkinir@gmail.coml

Termos para indexação:

Fixação de nitrogênio

Flora microbiana

Respiração do solo

Index terms:

Nitrogen fixation

Microbial flora

Soil respiration

Histórico do artigo:

Recebido em 22/11/2019

Aprovado em 19/08/2021

Publicado em 31/01/2022

\section{(c) (1) () $\Theta$

BY NC ND

Resumo - O aumento na deposição atmosférica de nitrogênio ao longo dos anos pode afetar a ciclagem biogeoquímica pela atuação da microbiota do solo. Objetivou-se avaliar a influência da deposição de $\mathrm{N}$ além das áreas das copas das árvores no efluxo de $\mathrm{C}-\mathrm{CO}_{2}$ do solo. Foram selecionadas quatro espécies arbóreas em campo aberto, sendo para cada uma destas selecionados três indivíduos e avaliado o efluxo de C- $\mathrm{CO}_{2}$ do solo sob suas copas com e sem a presença de nitrato de amônio $\left(\mathrm{NH}_{4} \mathrm{NO}_{3}\right)$. Essas foram comparadas com áreas de gramíneas (Paspalum notatum Flüggé). A adição do $\mathrm{NH}_{4} \mathrm{NO}_{3}$ induziu mudança na inclinação das retas que descrevem o efluxo de $\mathrm{C}-\mathrm{CO}_{2}$ do solo para áreas sob (105\%) e fora das copas das árvores (70\%). A área sob copa de Inga laurina foi a única a apresentar menor efluxo de $\mathrm{C}-\mathrm{CO}_{2}$. Conclui-se que a deposição anual de $\mathrm{N}$ inorgânico no estado do Rio de Janeiro $\left(9,6 \mathrm{~kg} \mathrm{~N} \mathrm{ha}^{-1}\right)$ intensifica o efluxo de $\mathrm{C}-\mathrm{CO}_{2}$ no solo e que espécies arbóreas com áreas de copa com maiores dimensões, como I. laurina, propiciam uma redução desse efluxo.

\section{Nitrogen deposition and tree canopy influence on soil $\mathrm{C}-\mathrm{CO}_{2}$ efflux}

\begin{abstract}
The increase in atmospheric nitrogen deposition over the years can affect biogeochemical cycling through the action of soil microbiota. The objective of this study was to evaluate the influence of $\mathrm{N}$ deposition beyond the tree canopy areas on soil $\mathrm{C}-\mathrm{CO}_{2}$ efflux. Four tree species were selected in the open field. For each species, three individuals were selected and the efflux of $\mathrm{C}-\mathrm{CO}_{2}$ from the soil under their canopies was evaluated with and without the presence of ammonium nitrate $\left(\mathrm{NH}_{4} \mathrm{NO}_{3}\right)$. They were compared with grass areas (Paspalum notatum Flüggé). The addition of $\mathrm{NH}_{4} \mathrm{NO}_{3}$ induced changes in the slope of the straight lines describing the soil $\mathrm{C}-\mathrm{CO}_{2}$ efflux to areas under (105\%) and outside the trees canopies (70\%). The area under Inga laurina canopy was the only one with lower $\mathrm{C}-\mathrm{CO}_{2}$ efflux. We concluded that Inorganic N deposition in the state of Rio de Janeiro $\left(9.6 \mathrm{~kg} \mathrm{~N} \mathrm{ha}^{-1}\right)$, intensifies the $\mathrm{C}_{-} \mathrm{CO}_{2}$ efflux in the soil and that tree species with bigger canopy areas such as I. laurina provide a reduction of this efflux.
\end{abstract}




\section{Introdução}

A produção de $\mathrm{CO}_{2}$ no interior do solo é influenciada pela temperatura, umidade do solo e também pelas práticas agrícolas/florestais, como por exemplo adubação, aração e gradagem (Lal, 2009; Wang et al., 2018). O fato dos microrganismos atuarem nos processos de gênese do solo, da decomposição de resíduos orgânicos, da ciclagem de nutrientes e na humificação da matéria orgânica (Mendes et al., 2011), justificam a importância da inclusão de indicadores microbiológicos nas avaliações de qualidade do solo (Tisdall \& Oades, 1982; Lopes et al., 2013; Mendes et al., 2015).

Nesse contexto, a respiração basal é um indicador da atividade microbiológica do solo no qual ocorre a oxidação biológica da matéria orgânica a $\mathrm{CO}_{2}$ pelos microrganismos aeróbios (Silva et al., 2013). Essa comunidade microbiana desempenha papel fundamental no ciclo biogeoquímico, sendo que mudanças nessa comunidade podem afetar diretamente o funcionamento do ecossistema (Mendes et al., 2017). Segundo Liu et al. (2016), a respiração do solo varia conforme a cobertura do dossel e os efeitos desta dependem das espécies de árvores. Assim, a redução da respiração do solo é propiciada pela menor temperatura e maior umidade do solo, sendo este fato mais expressivo em sistemas compostos por indivíduos com copas maiores e com maior biomassa (Murphy et al., 2008).

A qualidade do substrato depositado sobre o solo é um fator que exerce controle sobre a decomposição microbiana (Jagadamma et al., 2014). Os solos sob dossel de espécies vegetais fixadoras de $\mathrm{N}$ apresentam dinâmica distinta, quando comparados a áreas com espécies não-fixadoras (Zagatto et al., 2019). Essas espécies intensificam a ciclagem de nutrientes pela deposição diferenciada de N (Forrester et al., 2006), podendo assim potencializar a atividade microbiana e aumentar tanto a absorção de $\mathrm{CO}_{2}$ pelos produtores primários, como o sequestro de carbono no solo, minimizando o aumento das concentrações atmosféricas de $\mathrm{CO}_{2}$ (Resh et al., 2002; Fornara \& Tilman, 2012; Zagatto et al., 2019).

Os ecossistemas em todo o mundo estão recebendo quantidades crescentes de $\mathrm{N}$ por meio de atividades antropogênicas, o que pode representar impactos potencialmente importantes na dinâmica do $\mathrm{C}$ no solo (Ramirez et al., 2012). A cidade do Rio de Janeiro possui cerca de 20 milhões de habitantes, uma frota de veículos de aproximadamente 3 milhões, e é o segundo maior polo industrial do Brasil (Detran-RJ, 2019; IBGE, 2019). As emissões de $\mathrm{N}$ oriundas de fontes antropogênicas aumentam a deposição atmosférica de $\mathrm{N}$, desempenhando papel fundamental na poluição do ar em todo o mundo (Kuang et al., 2016). Essas causam impactos negativos à saúde e aos serviços do ecossistema, como a redução da biodiversidade (Clark \& Tilman, 2008) e acidificação global (Gao et al., 2013). Seu impacto na composição das chuvas e deposições atmosféricas tem sido estudado por décadas, com alterações mais importantes ocorrendo na vertente oceânica da cidade, em comparação à continental (Rodrigues et al., 2007; Souza et al., 2017). Contudo, seus efeitos sobre a vegetação e reservatórios de C ainda são escassos, principalmente no Brasil.

Esse trabalho parte da hipótese que as camadas superficiais de solos sob copas de árvores apresentam maiores efluxos de $\mathrm{C}-\mathrm{CO}_{2}$ para a atmosfera, em função da adição de $\mathrm{N}$ via deposição atmosférica, e estes ocorrem de forma diferenciada entre espécies que se associam a bactérias diazotróficas e aquelas que não se associam. A adição diferenciada de $\mathrm{N}$ via deposição atmosférica em áreas próximas do Rio de Janeiro tem sido constatada (Rodrigues et al., 2007; Souza et al., 2017), mas nenhum trabalho foi encontrado na literatura reportando a influência dessa deposição antropogênica sobre o $\mathrm{C}$ do solo em áreas próximas a esta cidade. Nesse sentido, o objetivo desse estudo foi avaliar o impacto da adição de $\mathrm{N}$, simulando entrada atmosférica, sobre o efluxo de $\mathrm{C}-\mathrm{CO}_{2}$ em solo coletado sob diferentes dosséis arbóreos e áreas adjacentes a eles, com predominância de gramíneas.

\section{Material e métodos}

\section{Caracterização da área}

$\mathrm{O}$ estudo foi conduzido na área da Universidade Federal Rural do Rio de Janeiro (UFRRJ), Seropédica,

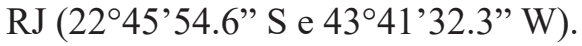

O clima predominante na região de Seropédica é Aw, segundo classificação de Köppen, caracterizado por verão quente e úmido e inverno seco. As médias anuais de temperatura e precipitação dos últimos 20 anos, são, respectivamente, $23,7^{\circ} \mathrm{C}, 1.275 \mathrm{~mm}$, com umidade relativa do ar de $69,3 \%$, obtidas da estação meteorológica da PESAGRO, RJ, a mais próxima ao local do experimento (Alonso et al., 2015). 
Parte considerável da área da UFRRJ foi drenada e os solos predominantes (Planossolos, Gleissolos e Argissolos) receberam a grama pernambuco (Paspalum notatum Flüggé) dada a rusticidade e efeito paisagístico. Ao longo dos anos, espécies arbóreas foram plantadas ou chegaram naturalmente a esses gramados. Foram selecionados dentro na área da UFRRJ três indivíduos (repetições) de quatro espécies de leguminosas arbóreas, estando isoladas em uma matriz de gramíneas. Foram selecionadas: duas espécies que se associam com bactérias fixadoras de N: Gliricidia sepium (Jacq.) Kunth ex Walp (gliricídia), Inga laurina (Sw.) Willd (ingá) e duas que não se associam a tais bactérias: Libidibia ferrea (Mart. ex Tul.) L. P. Queiroz (pau-ferro) e Poicianella pluviosa (DC.) L. P. Queiroz (sibipiruna). Nessas espécies, foram mensurados a altura total (HT) com auxílio de uma vara graduada, além do diâmetro a 1,30 m do solo (DAP) e área de copa, com auxílio da uma fita métrica.

$\mathrm{O}$ efluxo de $\mathrm{C}-\mathrm{CO}_{2}$ das amostras das camadas superficiais sob as copas das espécies selecionadas foi avaliado, conjuntamente às amostras sob gramínea $P$. notatum, localizadas a $15 \mathrm{~m}$ da extensão final do raio da copa das espécies arbóreas (Figura 1). Cada amostra de solo avaliada foi considerada uma parcela experimental, totalizando 24 parcelas (12 em áreas sob copa de árvores e 12 áreas de gramíneas).

Procedimento de amostragem e preparo da amostra de solo

As amostragens foram realizadas em outubro de 2017, usando parcelas em mini trincheiras $(10 \mathrm{~cm} \times 20 \mathrm{~cm} \times 20 \mathrm{~cm})$, com auxílio de uma pá reta. Foram coletadas por parcela experimental quatro amostras simples $(0-10 \mathrm{~cm})$ em forma de monólitos, formando uma amostra composta. As coletas, feitas sob copa de árvores, foram realizadas a uma distância de meio raio da copa da árvore em formato de cruz (Figura 1).

Após a coleta, as amostras compostas de solo passaram por uma peneira de malha de $4 \mathrm{~mm}$, para preservar a estrutura do solo. Após esse processo, foi feita uma limpeza manual para a retirada de resíduos de raízes e serapilheira. As amostras compostas foram acondicionadas em sacos plásticos e mantidas em caixa térmica para transporte para laboratório. No laboratório, subamostras (200 g) foram separadas para análises química e granulométrica, sendo posteriormente acondicionadas sob refrigeração $\left(4^{\circ} \mathrm{C}\right)$ por cinco dias, até a incubação das mesmas para avaliação do efluxo de $\mathrm{C}-\mathrm{CO}_{2}$.

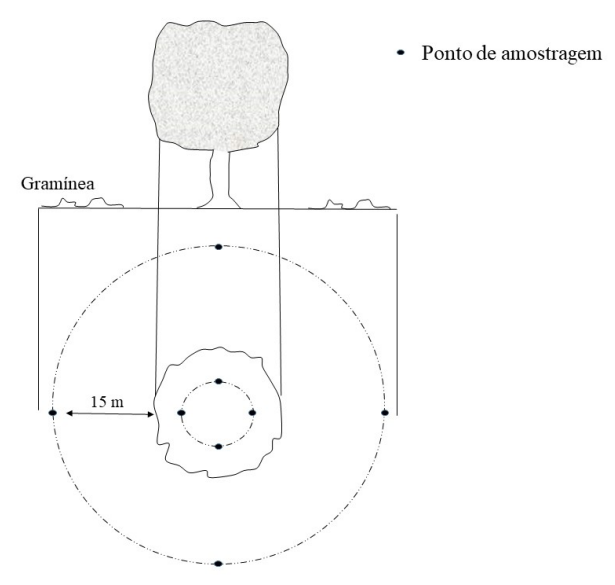

Figura 1. Croqui da localização dos pontos de coleta sob e fora da copa das árvores.

Figure 1. Location sketch of the sample points under and outside the tree canopy.

\section{Determinação do efluxo de $\mathrm{C}-\mathrm{CO}_{2}$}

Para determinação do efluxo de $\mathrm{C}-\mathrm{CO}_{2}$, foi utilizado o método adaptado de Jenkinson \& Powlson (1976). Cada amostra composta foi pesada (50 g) e colocada em recipiente (potes de $2 \mathrm{~L}$ ), sendo o valor de umidade ajustado a $60 \%$ da capacidade de campo. Frascos com 20 $\mathrm{mL}$ de $\mathrm{NaOH} 0,5 \mathrm{~mol} \mathrm{~L}^{-1}$ foram usados como armadilha (trap), para fixar o $\mathrm{CO}_{2}$ produzido durante a incubação, e outro com $20 \mathrm{~mL}$ de água destilada (previamente fervida com intuito da retirada do $\mathrm{CO}_{2}$ ), para manter as condições de umidade. Os potes foram fechados hermeticamente e cobertos com material plástico de cor escura, para simular a situação em campo. A temperatura média de incubação foi de $27^{\circ} \mathrm{C}$, por um período total de 10 dias.

A determinação do efluxo de $\mathrm{C}-\mathrm{CO}_{2}$ foi realizada após 24, 49, 96, 169 e 239 h do início do experimento. Em cada amostragem, o pote foi aberto por um período médio de 15 min, para retirada do frasco de $\mathrm{NaOH}$ com o auxílio de uma pinça. Nesse foi imediatamente adicionado $1 \mathrm{~mL}$ da solução de $\mathrm{BaCl}_{2} 50 \%$, para impedir que o $\mathrm{Na}_{2} \mathrm{CO}_{3}$ formado fosse decomposto em $\mathrm{NaOH}+$ $\mathrm{CO}_{2}$ durante a titulação. Foram acrescentadas 2 gotas 
do indicador fenolftaleína $1 \%$ para titulação com $\mathrm{HCl}$ padronizado a $0,4980 \mathrm{~mol} \mathrm{~L}^{-1}$ até o desaparecimento da cor rósea, sendo anotado o volume de $\mathrm{HCl}$ gasto na titulação. Após a titulação, procedeu-se aos cálculos da quantidade de $\mathrm{CO}$ liberado por grama de solo seco, durante o período determinado (Equação 1).

$$
\text { EFL (mg de C } \left.-\mathrm{CO}_{2} \mathrm{Kg}^{-1} \text { solo hora }{ }^{-1}\right)=\left(\frac{\left(\mathrm{V}_{\mathrm{b}}-\mathrm{V}_{\mathrm{a}}\right) \cdot \mathrm{M} \cdot 6 \cdot 1000}{\mathrm{P}_{\mathrm{S}}}\right) / \mathrm{T}
$$

Em que: $\mathrm{EFL}=$ efluxo de $\mathrm{C}-\mathrm{CO}_{2} ; \mathrm{Vb}(\mathrm{mL})=$ volume de ácido clorídrico gasto na titulação da solução controle (branco); $\mathrm{Va}(\mathrm{mL})=$ volume gasto na titulação da amostra; $\mathrm{M}=$ molaridade exata do ácido clorídrico; Ps $(\mathrm{g})=$ massa de solo seco e $\mathrm{T}$ = tempo de incubação da amostra (h).

Após 10 dias de incubação das amostras, os recipientes permaneceram abertos por um período de 15 dias, com intuito de realizar trocas gasosas com o ambiente e o estabelecimento do equilíbrio microbiano das amostras. Em seguida, aplicou-se em cada amostra 5,64 mg de nitrato de amônio $\left(\mathrm{NH}_{4} \mathrm{NO}_{3}\right)$ diluído em água destilada, sendo novamente determinado o efluxo de $\mathrm{C}-\mathrm{CO}_{2}$ pelo mesmo período de tempo.

A quantidade de $\mathrm{N}$ (na forma de nitrato de amônio) aplicada foi baseada no valor médio de deposição total de $\mathrm{N}$ inorgânico no estado do Rio de Janeiro, que é de $9,60 \mathrm{~kg} \mathrm{~N} \mathrm{ha}^{-1} \mathrm{ano}^{-1}$ (Souza et al., 2017), e na área do frasco $\left(19,64 \mathrm{~cm}^{2}\right)$ no qual as amostras de solo foram depositadas. Uma solução foi preparada com o objetivo de simular a deposição via precipitação.

\section{Análises estatísticas}

Assumiu-se o delineamento inteiramente casualizado, em que os dados de efluxo de $\mathrm{C}-\mathrm{CO}_{2}$, fertilidade e granulometria do solo foram analisados por meio da análise de variância. Quando necessário, as médias foram comparadas utilizando o teste de Tukey, adotandose o nível de $5 \%$ de probabilidade de erro.

Para avaliar a dinâmica de efluxo de $\mathrm{C}-\mathrm{CO}_{2}$ em função da aplicação de $\mathrm{N}$ e a copa das árvores, foi aplicado teste $t$ nos coeficientes angulares obtidos pela análise de regressão.

Todas as análises foram realizadas com o auxílio do software estatístico R (R Development Core Team, 2019).

\section{Resultados}

\section{Características gerais das áreas avaliadas}

Foram observadas características distintas quanto ao porte das espécies avaliadas (Tabela 1). Para Inga laurina foi verificada maior altura total (HT), diâmetro a 1,30 $\mathrm{m}$ do solo (DAP) e área de copa. As demais espécies não diferiram entre si $(\mathrm{p}>0,05)$ para as variáveis HT e DAP, sendo observado os menores valores. Contudo, em Gliricidia sepium foi verfiicada área de copa superior a Poicianella pluviosa e Libidibia ferrea.

A amostragem realizada nesse estudo buscou respeitar a posição das árvores na paisagem e a homogeneidade dos atributos do solo nos diferentes locais de coleta de amostras. Dessa forma, para todos os atributos avaliados, exceto $\mathrm{H}+\mathrm{Al}$, não foram observadas variações significativas entre os pontos de amostragem (Tabela 2).

Tabela 1. Valores médios de altura total (HT), diâmetro a 1,3 $\mathrm{m}$ do solo (DAP) e área de copa das espécies utilizadas em estudo de respiração do solo, em Seropédica, RJ.

Table 1. Average values of total height (TH), diameter at $1.3 \mathrm{~m}$ from the ground (DBH) and canopy area, of the species used in soil respiration study, Seropédica, Rio de Janeiro State, Brazil.

\begin{tabular}{cccc}
\hline Espécie & $\begin{array}{c}\text { HT* } \\
(\mathbf{m})\end{array}$ & $\begin{array}{c}\text { DAP* } \\
(\mathbf{c m})\end{array}$ & $\begin{array}{c}\text { Área da copa } \\
\left(\mathbf{m}^{\mathbf{2}}\right)\end{array}$ \\
\hline Gliricidia sepium & $8,5 \mathrm{~b}$ & $27,5 \mathrm{~b}$ & $38,9 \mathrm{~b}$ \\
Inga laurina & $11.8 \mathrm{a}$ & $68,4 \mathrm{a}$ & $88,8 \mathrm{a}$ \\
Poicianella pluviosa & $6,8 \mathrm{~b}$ & $20,7 \mathrm{~b}$ & $16,5 \mathrm{c}$ \\
Libidibia ferrea & $8,6 \mathrm{~b}$ & $25,9 \mathrm{~b}$ & $18,2 \mathrm{c}$ \\
\hline
\end{tabular}

Valores médios obtidos pela mensuração de três indivíduos (repetições). Médias seguidas por letras distintas, na coluna, diferem significativamente pelo teste de Tukey $(\mathrm{p}<0,05)$.

Efeito da deposição atmosférica de $N$ no efluxo de $\mathrm{C}-\mathrm{CO}_{2}$ do solo

A adição de $\mathrm{N}$ nas amostras de terra induziu uma mudança abrupta de inclinação das retas que descrevem o efluxo de $\mathrm{C}-\mathrm{CO}_{2}$ do solo, tanto dentro, quanto fora da área de influência da copa das árvores, com uma inclinação de aproximadamente $105 \%$ e $70 \%$ nas áreas sob e fora das copas, respectivamente (Figura 2). Contudo, não foi observada diferença significativa no local de amostragem (dentro e fora da copa). 
Tabela 2. Fertilidade e granulometria do solo nas áreas sob copa (sc) e fora da copa (fc) dos espécimes de Libidibia ferrea (Lfe), Gliricidia sepium (Gse), Inga laurina (Ila) e Poicianella pluviosa (Ppl) na profundidade de $0-10 \mathrm{~cm}$, em Seropédica, RJ.

Table 2. Soil fertility and granulometry in the canopy (sc) and non-canopy (fc) areas of Libidibia ferrea (Lfe), Gliricidia sepium (Gse), Inga laurina (Ila) e Poicianella pluviosa (Ppl) specimens at depth of $0-10 \mathrm{~cm}$, Seropédica, Rio de Janeiro State, Brazil.

\begin{tabular}{|c|c|c|c|c|c|c|c|c|c|c|c|c|}
\hline \multirow{2}{*}{ Áreas } & pH & $\mathrm{Ca}^{+2}$ & $\mathbf{M g}^{+2}$ & $\mathbf{A l}^{+3}$ & $\mathbf{N a}^{+}$ & $\mathbf{H}+\mathbf{A l}$ & $\mathbf{K}^{+}$ & $\mathbf{P}$ & \multirow{2}{*}{$\frac{\text { COT }}{\mathrm{g} \mathrm{kg}^{1}}$} & Areia & Silte & Argila \\
\hline & \multicolumn{2}{|l|}{ Água } & \multicolumn{4}{|c|}{ - } & \multicolumn{2}{|c|}{----mg kg-1-- } & & \multicolumn{3}{|c|}{ 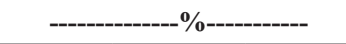 } \\
\hline sc-Lfe & 5,47 & 2,06 & 1,26 & 0,00 & 0,03 & $2,66 \mathrm{~b}$ & 89,93 & 9,97 & 13,10 & 43,30 & 10,57 & 46,13 \\
\hline fc-Lfe & 5,33 & 1,46 & 1,46 & 0,00 & 0,02 & $2,80 \mathrm{~b}$ & 63,86 & 6,67 & 12,67 & 36,36 & 12,79 & 50,85 \\
\hline sc-Gse & 4,90 & 1,13 & 1,13 & 0,20 & 0,03 & $3,36 \mathrm{~b}$ & 72,99 & 8,67 & 10,37 & 35,87 & 15,99 & 48,14 \\
\hline fc-Gse & 5,13 & 1,23 & 1,26 & 0,13 & 0,03 & $2,97 \mathrm{~b}$ & 67,77 & 7,33 & 12,43 & 37,98 & 12,17 & 49,85 \\
\hline sc-Ila & 4,80 & 2,13 & 1,20 & 0,40 & 0,05 & $5,63 \mathrm{a}$ & 91,23 & 7,33 & 14,60 & 25,67 & 16,83 & 57,50 \\
\hline fc-Ila & 5,57 & 1,36 & 1,10 & 0,00 & 0,03 & $2,33 \mathrm{~b}$ & 79,50 & 6,67 & 9,47 & 39,05 & 11,43 & 49,52 \\
\hline sc-Ppl & 5,07 & 1,40 & 1,45 & 0,13 & 0,03 & $3,13 \mathrm{~b}$ & 67,78 & 6,00 & 10,30 & 35,41 & 13,56 & 51,04 \\
\hline fc-Ppl & 5,37 & 1,16 & 1,10 & 0,00 & 0,02 & $2,76 \mathrm{~b}$ & 58,65 & 5,67 & 11,37 & 47,99 & 11,88 & 40,12 \\
\hline
\end{tabular}

$\mathrm{COT}=$ Carbono orgânico total. Médias seguidas por letras distintas, na coluna, diferem significativamente pelo teste de Tukey $(\mathrm{p}<0,05)$.

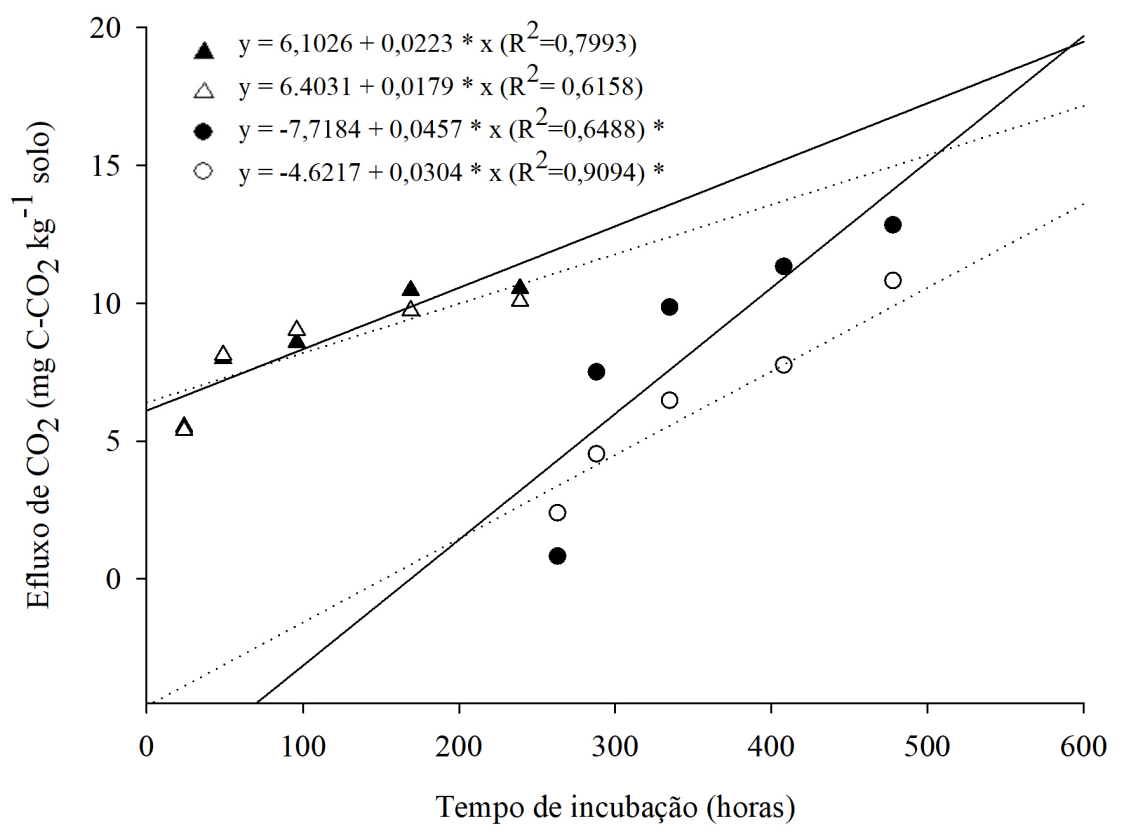

Figura 2. Efluxo de $\mathrm{CO}_{2}\left(\mathrm{mg} \mathrm{C}-\mathrm{CO}_{2} \mathrm{~kg}^{-1}\right.$ solo) do solo sob influência da copa de árvores e fora dessa influência e da deposição atmosfera de $\mathrm{N}$ simulada $\left(\mathrm{NH}_{4} \mathrm{NO}_{3}\right)$. Símbolo de triângulo refere-se ao efluxo antes da aplicação de $\mathrm{NH}_{4} \mathrm{NO}_{3}$ (preto, refere-se a área sob copa das árvores; branco, refere-se a área sob gramíneas). Símbolo de círculo refere-se ao efluxo após a aplicação de $\mathrm{NH}_{4} \mathrm{NO}_{3}$ (preto, refere-se a área sob copa das árvores; branco, refere-se a área sob gramíneas). Coeficientes angulares que apresentam " diferem estatisticamente pelo teste t quanto à deposição de $\mathrm{NH}_{4} \mathrm{NO}_{3}$. Linhas de regressão tracejadas representam as áreas sob gramíneas e as continuas representam as áreas sob copa de árvores.

Figure 2. $\mathrm{CO}_{2}$ efflux (mg C- $\mathrm{CO}_{2} \mathrm{~kg}^{-1}$ soil) from soil under and outside tree canopy and simulated $\mathrm{N}$ atmosphere deposition $\left(\mathrm{NH}_{4} \mathrm{NO}_{3}\right)$. Triangle symbol refers to efflux before $\mathrm{NH}_{4} \mathrm{NO}_{3}$ application (black, refers to area under tree canopy; white, refers to area under grasses). Circle symbol refers to efflux after $\mathrm{NH}_{4} \mathrm{NO}_{3}$ application (black, refers to area under tree canopy; white, refers to area under grasses). Angular coefficients presenting * differ statistically by t-test for $\mathrm{NH}_{4} \mathrm{NO}_{3}$ deposition. Dotted regression lines refer to areas under grasses. Continuous regression lines refer to areas under canopies. 
Efeito das espécies sobre o efluxo de $\mathrm{C}-\mathrm{CO}_{2}$ do solo

Somente para o material coletado na área de I. laurina foi verificada diferença do efluxo de $\mathrm{C}-\mathrm{CO}_{2}$ sob e fora da copa. $\mathrm{Na}$ ausência de efeito do $\mathrm{N}$ mineral aplicado (0-239 dias) na área das espécies fixadoras de $\mathrm{N}$ foi verificada maior similaridade nos efluxos de $\mathrm{C}-\mathrm{CO}_{2}$ que as não fixadoras, apesar de não ter sido constatada diferenças nos totais emitidos no material coletado nas áreas dessas espécies (Figura 3), padrão diferenciado do observado quando foi adicionado $\mathrm{N}$ às amostras. As amostras coletadas nas áreas das duas espécies não fixadoras de $\mathrm{N}_{2}$ foram influenciados da mesma forma pela adição de $\mathrm{N}$, sendo observadas taxas de efluxo de $\mathrm{C}-\mathrm{CO}_{2}$ similares. Entre as fixadoras, e no material coletado na área de G. sepium, foi verificado efluxo de $\mathrm{C}-\mathrm{CO}_{2}$ superior àquele observado para as amostras oriundas da área de I. laurina, quando a deposição de $\mathrm{N}$ foi realizada.
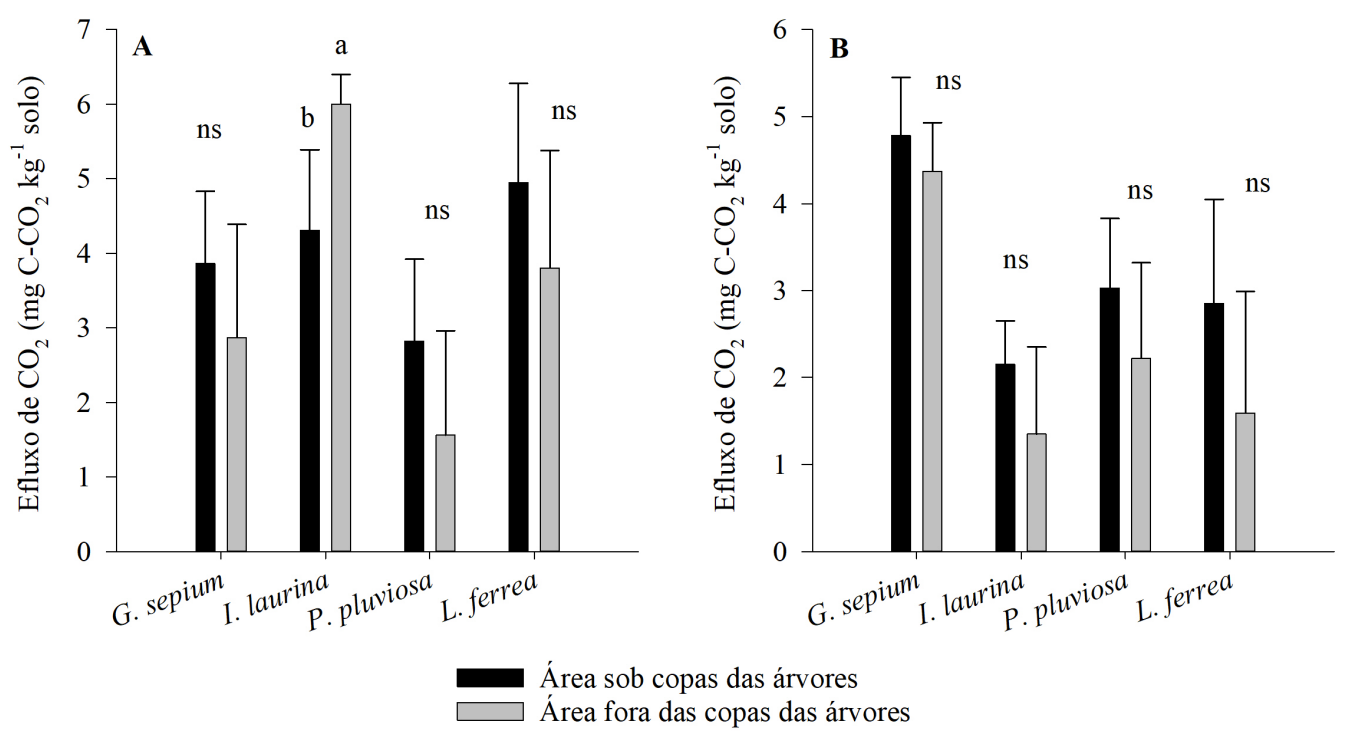

Figura 3. Efluxo de $\mathrm{C}-\mathrm{CO}_{2}$ acumulado ( $\mathrm{mg} \mathrm{C}-\mathrm{CO}_{2} \mathrm{~kg}^{-1}$ solo) do solo em áreas sob e fora das copas sem (A) e com (B) a aplicação de nitrato de amônio $\left(\mathrm{NH}_{4} \mathrm{NO}_{3}\right)$ em função de espécimes arbóreas de Gliricidia sepium, Inga laurina, Poicianella pluviosa e Libidibia ferrea. Os valores seguidos pela mesma letra não diferem significativamente pelo teste $t$.

Figure 3. Accumulated $\mathrm{C}-\mathrm{CO}_{2}$ efflux (mg C- $\mathrm{CO}_{2} \mathrm{~kg}^{-1}$ soil) from soil under and outside canopy areas without (A) and with (B) ammonium nitrate $\left(\mathrm{NH}_{4} \mathrm{NO}_{3}\right)$ application as a function of Gliricidia sepium tree specimens, Inga laurina, Poicianella pluviosa and Libidibia ferrea. The values followed by the same letter do not differ significantly by the t-test.

\section{Discussão}

A dose de $\mathrm{N}$ usada nesse estudo $\left(9,6 \mathrm{~kg} \mathrm{ha}^{-1}\right)$ interferiu na dinâmica das comunidades microbianas do solo, estimulando-as a decompor matéria orgânica, especialmente nas amostras coletadas sob a copa das árvores. A lavagem da copa das árvores (throughfall) aumenta a entrada de fontes lábeis de $\mathrm{C}$ e nutrientes (Parker, 1983). Esse padrão demonstra ainda que, em longo prazo, as deposições agudas de $\mathrm{N}$ podem implicar em reduções significativas dos estoques de $\mathrm{C}$ do solo em ambientes arborizados, caso a entrada de $\mathrm{C}$ não seja compensada. Os resultados encontrados também corroboram com os padrões verificados por Maaroufi et al. (2015). Os autores constataram que após 16 anos simulando a deposição de $\mathrm{NH}_{4} \mathrm{NO}_{3}$, baixas doses (como nesse estudo) provocaram aumento do efluxo de $\mathrm{CO}_{2}$ em área de floresta tardia ( $\sim 120$ anos). Janssens et al. (2010), afirmam ainda que a quantidade de $\mathrm{N}$ admitido para que ocorra o aumento do efluxo de $\mathrm{C}-\mathrm{CO}_{2}$ no solo 
está diretamente relacionado ao maior teor de lignina do material aportado. Assim, a adição de $\mathrm{N}$ tende a acelerar o efluxo em áreas com resíduos vegetais de baixa recalcitrância.

$\mathrm{O}$ fato da área sob copa de Inga laurina ser a única a apresentar um efluxo de $\mathrm{C}-\mathrm{CO}_{2}$ inferior à área fora da copa está diretamente associado à sua maior área de copa $\left(88,8 \mathrm{~m}^{2}\right)$. A respiração do solo varia conforme a cobertura do dossel (Liu et al., 2016). Segundo Murphy et al. (2008), sistemas dotados de indivíduos com copas maiores reduzem a respiração do solo, devido a menor temperatura e maior umidade propiciados pelo sistema. Portanto, sugere-se que as copas de Gliricidia sepium, Poicianella pluviosa e Libidibia ferrea não apresentam dimensões grandes o suficiente para promover a redução do efluxo de $\mathrm{C}-\mathrm{CO}_{2}$.

Outro fator que pode explicar o efluxo de $\mathrm{C}-\mathrm{CO}_{2}$ inferior na área sob copa de I. laurina é a qualidade de matéria orgânica do solo. A quantidade de C lábil no solo, facilmente mineralizável, pode variar espacialmente, principalmente em função do padrão de deposição e qualidade do material vegetal (Gholz et al., 2000). Como verificado por Ritll et al. (2015), a relação $\mathrm{C}: \mathrm{N}$ pode não ser um bom preditor da velocidade de decomposição da matéria orgânica do solo. Segundo esses autores, a proporção do $\mathrm{C}$ aromático e alifático dos resíduos adicionados ao solo são os maiores preditores da evolução do $\mathrm{CO}_{2}$ do solo em comparação à própria relação $\mathrm{C}: \mathrm{N}$ do material adicionado. Mesmo sendo espécies leguminosas fixadoras de $\mathrm{N}_{2}$, as comunidades microbianas do solo de G. sepium e de I. laurina diferiram no padrão de respiração e efluxo de C. Embora os valores de COT do solo na área sob copa de I. laurina tenham sido maiores que sob G. sepium, as emissões de C- $\mathrm{CO}_{2}$ foram superiores nessa área, quando da deposição de N. Uma possível explicação para esse padrão pode ser atribuída à qualidade do $\mathrm{C}$ do solo, considerando que o $\mathrm{N}$ não deve ser limitante na área de ocorrência dessas espécies.

Solos de pastagens, por outro lado, podem apresentar atividade microbiana elevada pela biomassa e volume de raízes que exploram o solo, e suas interações micorrízicas intensas que funcionam como bombas de C para o solo (Souza et al., 2010). Em estudo onde foi avaliado o efluxo em diferentes coberturas do solo, Jiang et al. (2011) encontraram as maiores taxas de respiração em áreas com menor ou nenhuma presença de espécie arbórea. Segundo os autores, em função da maior biomassa de raízes das gramíneas em comparação com espécies arbóreas. Embora nesse estudo a respiração autotrófica não tenha sido contabilizada, o resultado da sua presença foi computado ao final do período de incubação.

\section{Conclusões}

A deposição de $\mathrm{N}$ inorgânico no estado do Rio de Janeiro $\left(9,6 \mathrm{~kg} \mathrm{~N} \mathrm{ha}^{-1}\right)$ intensifica o efluxo de $\mathrm{C}-\mathrm{CO}_{2}$ no solo em até $105 \%$, evidenciando que alterações na atividade microbiana reduzem os estoques de $\mathrm{C}$ do solo, caso as entradas de $\mathrm{C}$ não sejam compensatórias.

Espécies arbóreas com áreas de copa com maiores dimensões, como I. laurina, propiciam uma redução do efluxo de $\mathrm{C}-\mathrm{CO}_{2}$.

Árvores isoladas em áreas de pastagens (como nesse estudo), prestam diversos serviços ambientais, além de conforto térmico propiciado para animais (sistema silvipastoril), que levam a melhoria de parâmetros produtivos e reprodutivos dos rebanhos. Adicionalmente, as árvores podem aumentar a interceptação das chuvas e a infiltração de água, mitigando o efeito erosivo. As espécies escolhidas para comporem paisagens rurais são diversas, e por isso é interessante que haja esforço no sentido de avaliá-las quanto à conservação do $\mathrm{C}$ do solo.

\section{Agradecimentos}

O presente trabalho foi realizado com apoio da Coordenação de Aperfeiçoamento de Pessoal de Nível Superior - Brasil (CAPES) - Código de Financiamento 001.

\section{Referências}

Alonso, J. K. et al. Aporte de serapilheira em plantio de recomposição florestal em diferentes espaçamentos. Ciência Florestal, v. 25, p. 1-11, 2015. http://dx.doi.org/10.5902/1980509817439.

Clark, C. M. \& Tilman, D. Loss of plant species after chronic lowlevel nitrogen deposition to prairie grasslands. Nature, v. 451, p. 712-715, 2008. http://dx.doi.org/10.1038/nature06503.

Detran-RJ. Departamento de Trânsito do Estado do Rio de Janeiro. Estatística da Frota de veículos do município do Rio de Janeiro. Disponível em: http://detran.rj.gov.br/_estatisticas.veiculos/02.asp. Acesso em: 21 nov. 2019.

Fornara, D. A. \& Tilman, D. Soil carbon sequestration in prairie grasslands increased by chronic nitrogen addition. Ecology, v. 93, p. 2030-2036, 2012. http://dx.doi.org/10.1890/12-0292.1. 
Forrester, D. I. et al. Mixed-species plantations of Eucalyptus with nitrogen-fixing trees: a rewiew. Forest Ecology and Management, v. 233, p. 211-230, 2006. http://dx.doi.org/10.1016/j. foreco.2006.05.012.

Gao, W. L. et al. Effects of simulated atmospheric nitrogen deposition on inorganic nitrogen content and acidification in a cold-temperate coniferous forest soil. Acta Ecológica Sinica, v. 33, p. 114-121, 2013. http://dx.doi.org/10.1016/j.chnaes.2013.01.008.

Gholz, H. L. et al. Long-term dynamics of pine and hardwood litter in contrasting environments: toward a global model of decomposition. Global Change Biology, v. 6, p. 751-765, 2000. http://dx.doi. org/10.1046/j.1365-2486.2000.00349.x.

IBGE. Instituto Brasileiro de Geografia e Estatística. Panorama das cidades brasileira. Disponível em: https://cidades.ibge.gov.br/brasil/ rj/rio-de-janeiro/panorama. Acesso em: 21 nov. 2019.

Jagadamma, S. et al. Substrate quality alters microbial mineralization of added substrate and soil organic carbon. Biogeosciences, v. 11, p. 4451- 4482, 2014. http://dx.doi.org/10.5194/bg-11-4665-2014.

Janssens, I. A. et al. Reduction of forest soil respiration in response to nitrogen deposition. Nature Geoscience, v. 3, p. 315-322, 2010. http://dx.doi.org/10.1038/ngeo844.

Jenkinson, D. S. \& Powlson, D. S. The effects of biocidal treatments on metabolism in soil. V. Method for measuring soil biomass. Soil Biology and Biochemistry, v. 8, p. 209-213, 1976. http://dx.doi. org/10.1016/0038-0717(76)90005-5.

Jiang, L. et al. Plant species effects on soil carbon and nitrogen dynamics in a temperate 216 steppe of northern China. Plant and Soil, v. 346, p. 331-347, 2011. http://dx.doi.org/10.1007/s11104011-0822-y.

Kuang, F. et al., Wet and dry nitrogen deposition in the central Sichuan Basin of China, Atmospheric Environment, v. 143, p. 39-50, 2016. http://dx.doi.org/10.1016/j.atmosenv.2016.08.032.

Lal, R. Challenges and opportunities in soil organic matter research. European Journal of Soil Science, v. 60, p. 158-169, 2009. http:// dx.doi.org/10.1111/j.1365-2389.2008.01114.x.

Liu, Q. et al. Temperature sensitivity of soil respiration to nitrogen fertilization: varying effects between growing and non-growing seasons. PLoS One, v. 11, e:0168599, 2014. http://dx.doi. org/10.1371/journal.pone.0168599.

Lopes, A. A. C. et al. Interpretation of microbial soil indicators as a function of crop yield and organic carbon. Soil Science Society of America Journal, v. 77, p. 461-472, 2013. http://dx.doi.org/10.2136/ sssaj2012.0191.

Maaroufi, N. I. et al. Anthropogenic nitrogen deposition enhances carbon sequestration in boreal soils. Global Change Biology, v. 21, p. 3169-3180, 2015. http://dx.doi.org/10.1111/gcb.12904.

Mendes, I. C. et al. Bioindicadores de qualidade de solo: dos laboratórios de pesquisa para campo. Cadernos de Ciência e Tecnologia, v. 32, p. 185-203, 2015.

Mendes, I. C. et al. Microbiologia do solo e sustentabilidade de sistemas agrícolas. In: Falieiro, F. G. et al. (ed.). Biotecnologia: estado da arte e aplicações na agropecuária. Brasília, DF: Embrapa, 2011. p. 219-244.
Mendes, R. et al. Efeito do aquecimento global sobre a comunidade microbiana do solo. In: Bettiol, W. et al. (ed.). Aquecimento global e problemas fitossanitários. Brasília, DF: Embrapa, 2017. p. 177-203.

Murphy, M. et al. Linking tree biodiversity to belowground process in a young tropical plantation: Impacts on soil $\mathrm{CO}_{2}$ flux. Forest Ecology and Management, v. 255, p. 2577-2588, 2008. http:// dx.doi.org/10.1016/j.foreco.2008.01.034.

Parker, G. G. Throughfall and stemflow in the forest nutrient cycle. Advances in Ecological Reserarch, v. 13, p. 58:120, 1983.

Ramirez, K. S. et al. Consistent effects of nitrogen amendments on soil microbial communities and processes across biomes. Global Change Biology, v. 18, p. 1918-1927, 2012. http://dx.doi. org/10.1111/j.1365-2486.2012.02639.x.

R Development Core Team. R: a language and environment for statistical computing. Vienna: R Foudation for Statistical Computing, 2019. Disponível em: http://www.R-project.org; 2019.

Resh, S. C. et al. Greater soil carbon sequestration under nitrogenfixing trees compared with Eucalyptus species. Ecosystems, v. 5, p. 217-231, 2002. http://dx.doi.org/10.1007/s10021-001-0067-3.

Ritll, T. F. et al. Negative priming of native soil organic carbon mineralization by oilseed biochars of contrasting quality. European Journal of Soil Science, v. 66, p. 714-721, 2015. https://doi. org/10.1111/ejss.12257.

Rodrigues, R. A. R. et al. Aporte atmosférico de amônio, nitrato e sulfato em área de Floresta Ombrófila Densa Montana na Serra dos Órgãos, RJ. Revista Química Nova, v. 30, 2007. http://dx.doi. org/10.1590/S0100-40422007000800009.

Silva, J. M. et al. Mineralização de vermicompostos estimada pela respiração microbiana. Revista Verde, v. 8, p. 132-135, 2013.

Souza, E. D. et al. Biomassa microbiana do solo em sistema de integração lavoura-pecuária em plantio direto, submetido a intensidades de pastejo. Revista Brasileira de Ciência do Solo, v. 34, p. 79-88, 2010. http://dx.doi.org/10.1590/S010006832010000100008.

Souza, P. A. et al. Deposições atmosféricas úmida, seca e total de nitrogênio inorgânico dissolvido no estado do Rio de Janeiro. Revista Virtual de Química, v. 9, p. 2052-2066, 2017. https://doi. org/10.21577/1984-6835.20170122.

Tisdall, J. M. \& Oades, J. M. Organic matter and water-stable aggregates in soils. European Journal of Soil Science, v. 33, p. 141-163, 1982. https://doi.org/10.1111/j.1365-2389.1982.tb01755.x.

Wang, Y. et al. Effects of forest regeneration practices on the flux of soil $\mathrm{CO}_{2}$ after clear-cutting in subtropical China. Journal of Environmental Management, v. 212, p. 332-339, 2018. https:// doi.org/10.1016/j. jenvman.2018.02.038.

Zagatto, M. R. G. et al. Interactions between mesofauna, microbiological and cheminal soil attributes in pure and intercropped Eucalyptus and Acacia mangium plantations. Forest Ecology and Management, v. 433, p. 240-247, 2019. http://dx.doi.org/10.1016/j. foreco.2018.11.008. 